\title{
The Dynamics of the Nucleation, Growth and Termination of Single-Walled Carbon Nanotubes from in situ Raman Spectroscopy During Chemical Vapor Deposition
}

\author{
Paul Finnie ${ }^{1,2}(\bowtie)$, Andrew Li-Pook-Than ${ }^{1,2}$, and Jacques Lefebvre ${ }^{1}$ \\ ${ }^{1}$ Institute for Microstructural Sciences, National Research Council Canada, Building M-50, 1200 Montreal Road, Ottawa, ON, K1A \\ 0R6, Canada \\ ${ }^{2}$ Department of Physics, University of Ottawa, 150 Louis Pasteur, Ottawa, ON K1N 6N5, Canada \\ Received: 19 April 2009 / Revised: 19 June 2009 / Accepted: 11 August 2009 \\ CTsinghua University Press and Springer-Verlag 2009. This article is published with open access at Springerlink.com
}

\begin{abstract}
The dynamics of the chemical vapor deposition (CVD) of single-walled carbon nanotubes (SWNTs) is extracted experimentally using in situ Raman spectroscopy. Nanotubes are grown using a thin film cobalt catalyst and an ethanol precursor in a miniature hot walled reactor with optical access. Raman spectra at room temperature and at the growth temperature are compared for two growth temperatures. The evolution of the G-band, D-band, and radial breathing mode (RBM) is tracked at the growth temperature with time resolution of a few seconds. There are three identifiable phases in the evolution of the Raman signal intensity: an initial exponential increasing phase, a linear growth phase, and a saturation phase. In situ optical spectroscopy thus enables the study of nucleation, steady growth, and deactivation processes to be investigated separately in real time. The evolution curves for all bands (G, D, and RBM), when scaled, collapse onto the same curve, to within experimental uncertainty.
\end{abstract}

\section{KEYWORDS}

Single-walled carbon nanotube, chemical vapor deposition, Raman spectroscopy, nucleation, termination

As carbon nanotubes continue to increase in scientific and technological importance, it becomes more and more important to understand the process of nanotube synthesis and to better control the end product of the synthesis. Chemical vapor deposition (CVD) is probably the leading method of synthesizing carbon nanotubes. For most research directions and most applications it is no longer sufficient just to produce single-walled carbon nanotubes (SWNTs). Researchers would like reproducible, precision control of the growth product, and detailed information about the growth process, preferably in real time. To these ends, more and more researchers are turning to in situ methods to study synthesis. Raman spectroscopy is extremely well developed as a characterization tool for nanotubes and other nanocarbons, and it is also compatible with the high temperatures used for SWNT CVD. Thus, it is natural to employ it as an in situ tool for the study of SWNT growth.

Address correspondence to Paul.Finnie@nrc.ca 
There have already been several reports of in situ Raman spectroscopy of CVD growth of SWNTs. An early spectroscopic study reported a linear increase in the G band signal during CVD using a zeolite/ Fe -Co-based catalyst and ethanol vapor as the carbon source [1]. In a combined Raman spectroscopy-global Raman imaging (GRI) study, we were able to detect SWNTs as they formed in the reactor and to trace out the $\mathrm{D}$ and $\mathrm{G}$ band evolution [2]. This was for thin film metal catalysts on silicon dioxide and using ethanol vapor as the carbon source. More recently in situ Raman spectroscopy of radial breathing mode (RBM), $\mathrm{D}$ and $\mathrm{G}$ bands, has been reported for an iron thin film catalyst on alumina, using ethylene as the carbon source, with a micro-heating approach [3]. In that case, however, spectra were taken only after cooling to room temperature. In a very recent study using $\mathrm{Ni}$ and Co thin film catalysts and ethanol vapor as the carbon source, the absolute $G$ band intensity was tracked as a function of time for various temperatures and pressures [4]. The intensity of the G band was observed to increase linearly and saturate, and initial growth rates and decay times were extracted.

There is a large body of work applying other in situ diagnostics to CVD growth of both SWNTs and multiwalled carbon nanotubes (MWNTs). For a more general discussion see Ref. [2]. Of special importance here are recent studies of dense forests of nanotubes, which are especially well suited to in situ observation. Such forests have been monitored by in situ reflection [5-7], in situ absorption [8], displacement sensing [9], and simple video [10-12]. Also, of interest here are electron beam studies of SWNT growth rates [13] and also microbalance experiments that have been used to track the MWNT carbon yield as a function of time [14].

\section{Experimental}

Samples were $0.5 \mathrm{~mm}$ thick silicon with $1.0 \mu \mathrm{m}$ thermal oxide, cleaved to approximately $5 \mathrm{~mm}$ by $5 \mathrm{~mm}$ pieces. An approximately $1.0 \mathrm{~nm}$ thick Co metal catalyst was deposited by e-beam evaporation. Samples were loaded into the reactor and purged (Ar 98\% $\mathrm{H}_{2} 2 \%$, $\sim 80$ standard cubic centimetres per minute $(\mathrm{sccm}))$ at room temperature and again at $200{ }^{\circ} \mathrm{C}$. Samples were rapidly heated to the growth temperature $\left(100{ }^{\circ} \mathrm{C} / \mathrm{min}\right)$. The flow rate was reduced to $2 \mathrm{sccm}$, and the gas diverted through a room temperature ethanol bubbler for a total of 20 min. After this, the bubbler was again bypassed, and the reactor purged at the growth temperature, and finally the sample was cooled to room temperature.

Raman spectra were obtained from a home built Raman spectroscopy/GRI system [2, 15]. The setup is illustrated in Fig. 1. Here, illumination was with a $532 \mathrm{~nm}$ laser. In contrast to conventional Raman spectroscopy, we use a highly defocused laser spot $(\sim 100 \mu \mathrm{m}$ diameter $)$ with a relatively high power $(\sim 0.3 \mathrm{~W})$ incident on the sample. Because of the high power, a strong signal was easily obtained. However, the power density $\left(\sim 30 \mu \mathrm{W} / \mu \mathrm{m}^{2}\right)$ was several orders of magnitude lower than in the Raman studies with

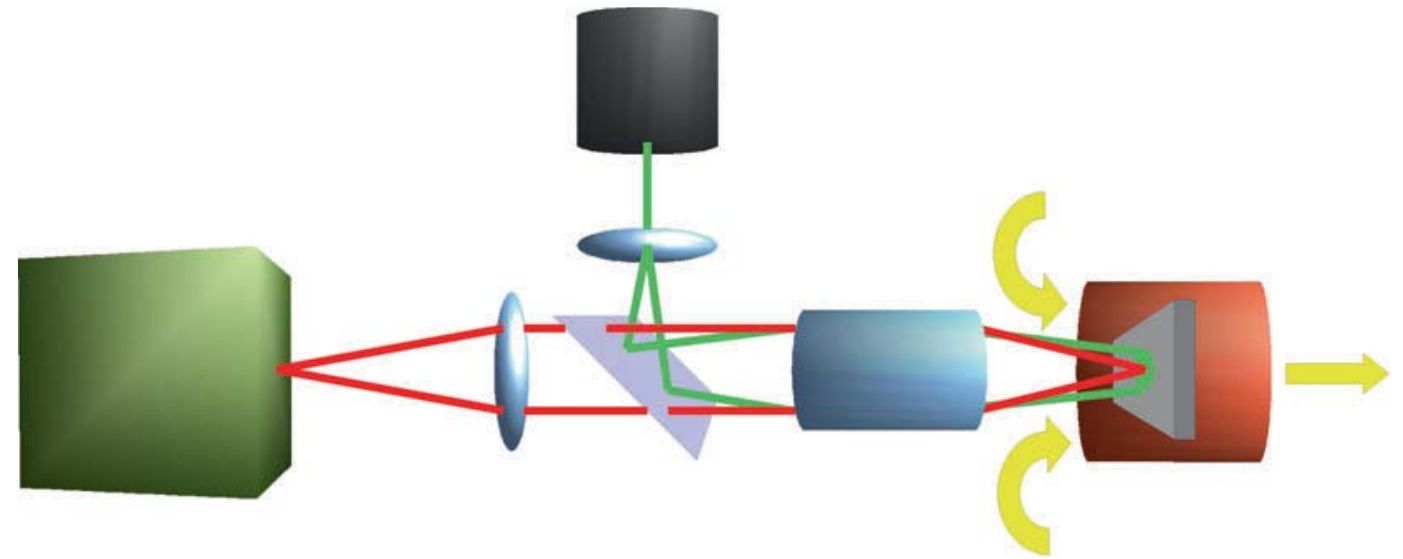

Figure 1 Schematic Raman and chemical vapor deposition setup. The sample (gray) is illuminated by a $532 \mathrm{~nm}$ defocused laser beam (green). The Raman signal (red) is collected by a microscope objective (blue) and focused onto a spectrometer (olive). The illumination spotsize is $\sim 100 \mu \mathrm{m}$ in diameter. Ethanol vapor and carrier gas (yellow arrows) flow through a miniature hot walled reactor (orange) 
focused spots, and so local heating was negligible. For comparison, Ref. [4] used $12 \mathrm{~mW}$ in a $1 \mu \mathrm{m}$ diameter spot, equivalent to a much higher power density of $12 \mathrm{~mW} / \mu \mathrm{m}^{2}$. The authors of that study provided convincing evidence that heating was negligible for their particular experimental conditions. However, in our experience such power densities are enough to ignite nanotube forests in ambient air, indicating that significant heating can occur for such high power densities, and this motivates us to use much lower power densities. Of course, since a high total power was used in our case, this will amount to a larger total heating of the sample, but this is distributed over a large area, making it locally negligible.

The reactor body for this work was a commercial mini-hot walled reactor (Linkam CCR1000) in which gas flows into and through a cylindrical ceramic heating element which is approximately $0.7 \mathrm{~mm}$ in diameter. The optical access is from the end of the cylinder, and the sample sits in the middle of the furnace. The temperature is measured with a thermocouple.

The signal was collected by a $20 \times$ microscope objective (numerical aperture 0.60), filtered and dispersed by a 1200 line $/ \mathrm{mm}$ grating in a 0.25 m spectrometer using 100 micron slit width. Spectroscopic data were collected by a thermoelectric (TE) cooled CCD detector. With a beam splitter, as in Ref. [2], GRI data and Raman spectra were obtained simultaneously. However, because the catalyst was uniform, and the nanotube density very high (roughly $\sim 10$ to $\sim 100$ nanotubes $/ \mu \mathrm{m}^{2}$, as revealed by SEM imaging) the Raman image was uniform and contained no significant spatial information other than the illumination profile over $100 \mu \mathrm{m}$ scale distances. Therefore, we turn our attention only to spectroscopy and the time evolution of the Raman spectrum.

\section{Results and discussion}

In this paper we illustrate the results of growths at two different temperatures, for which the Raman spectra are significantly different. The results of a "low temperature" run at $725^{\circ} \mathrm{C}$ will be shown as well as a "high temperature" run at $875^{\circ} \mathrm{C}$. As is commonly observed for thermal CVD, significant SWNT yields are only observed above a minimum temperature, although the minimum temperature is not generally agreed upon. In our case, which may be specific to the present catalyst or precursor, significant SWNT yields (as determined by the frequent detection of RBM modes in the Raman spectra) were only obtained above $\sim 650{ }^{\circ} \mathrm{C}$.

Figure 2 shows the full Raman spectra for the low temperature $\left(725^{\circ} \mathrm{C}\right)$ case. A $15 \mathrm{~s}$ integration was used for each spectrum and all spectra are plotted on the same scale. Figure 2(a) shows the Raman spectra of the sample at room temperature, before the experiment. All observed peaks are substraterelated, the most notable being silicon first order and second order peaks (near $500 \mathrm{~cm}^{-1}$ and $1000 \mathrm{~cm}^{-1}$ ) and another substrate related peak at $\sim 300 \mathrm{~cm}^{-1}$. Figure 2(b) shows the Raman spectra of the sample at 725 ${ }^{\circ} \mathrm{C}$, under purge gas, before the ethanol carbon source is supplied. The first and second order silicon peaks are downshifted, as expected, and a faint blackbody tail is visible at high wavenumber. Figure 2(c) shows the Raman spectra after CVD growth, but still at the $725{ }^{\circ} \mathrm{C}$ growth temperature. Both $\mathrm{D}\left(\sim 1300 \mathrm{~cm}^{-1}\right)$ and G bands $\left(\sim 1580 \mathrm{~cm}^{-1}\right)$ are clearly visible against a relatively flat background. Comparing Figs. 2(b) and 2 (c) in the RBM region $\left(<450 \mathrm{~cm}^{-1}\right)$ one can clearly see the emergence of at least one RBM peak, just to the left of the substrate-related peak. At least one additional peak is also present as a small shoulder, but this is somewhat difficult to discern clearly. Figure 2(d) shows the Raman spectra after cooling to room temperature. All the carbon-related peaks become sharper after cooling. Most notably, many more RBM modes are visible and they have much higher intensities.

The relative weakness of the RBM signal at high temperature probably stems from the fact that the $\mathrm{RBM}$ resonance in energetic terms is only of the order of $20 \mathrm{meV}$, i.e., approximately the thermal energy $\mathrm{kT}$ at room temperature. By comparison, the $\mathrm{D}$ and $\mathrm{G}$ bands, while sharper, are much less changed in intensity, which can be understood because they are much stiffer $(\sim 0.2 \mathrm{eV}$, i.e., approximately $\mathrm{kT}$ at $3000 \mathrm{~K})$. The $\mathrm{D}$ to $\mathrm{G}$ ratio is slightly lower at room temperature, but the most significant change is that the $\mathrm{G}$ band becomes much sharper and multiple G- 


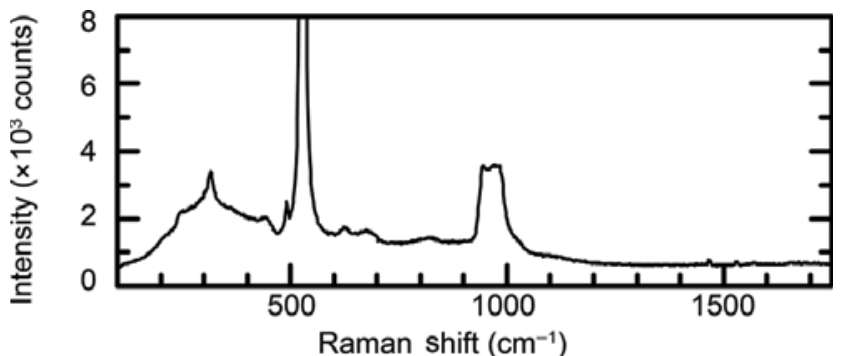

(a)

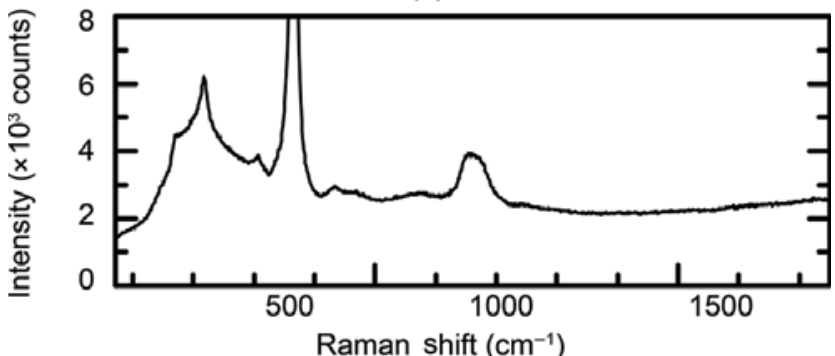

(b)

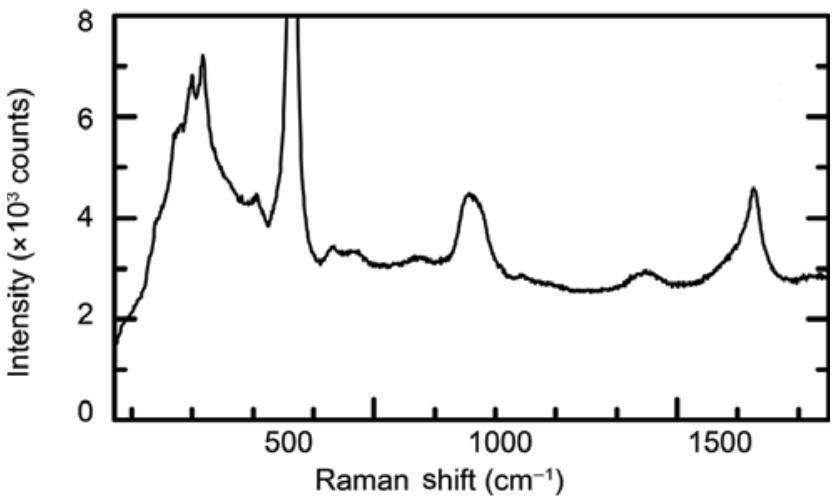

(c)

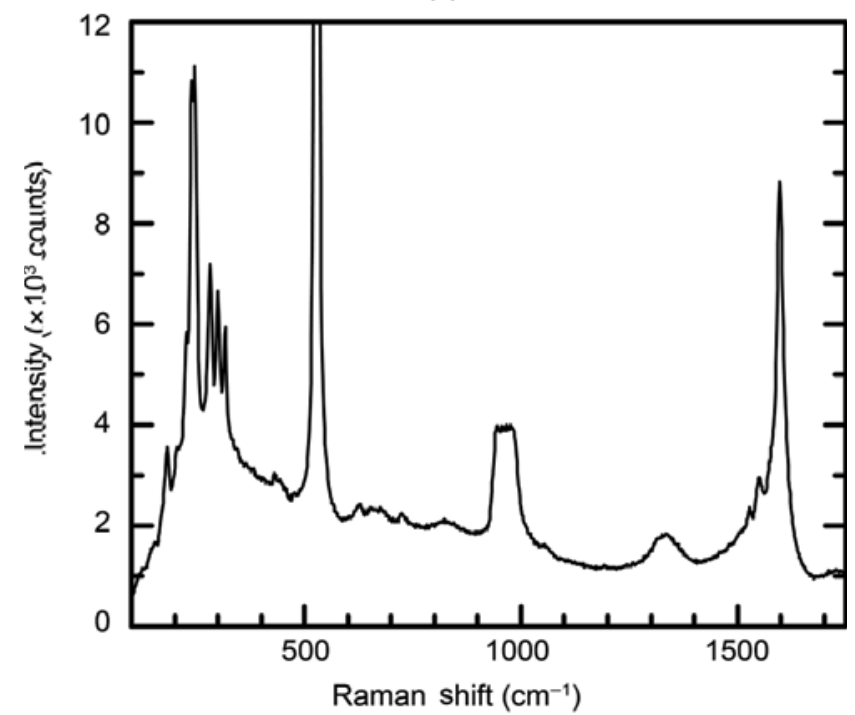

(d)

Figure 2 Raman spectra, pre- and post-growth at $725^{\circ} \mathrm{C}$ : (a) Room temperature, pre-growth; (b) $725^{\circ} \mathrm{C}$, pre-growth; (c) $725^{\circ} \mathrm{C}$, postgrowth; (d) room temperature, post-growth. All spectra are plotted on the same scale peaks, corresponding to different nanotube diameters (since the G- peak is diameter dependent) are clearly visible at room temperature, whereas one G- peak is perhaps barely discernible at high temperature.

Figure 3 shows the corresponding Raman spectra at high temperature $\left(875^{\circ} \mathrm{C}\right)$. At this temperature,

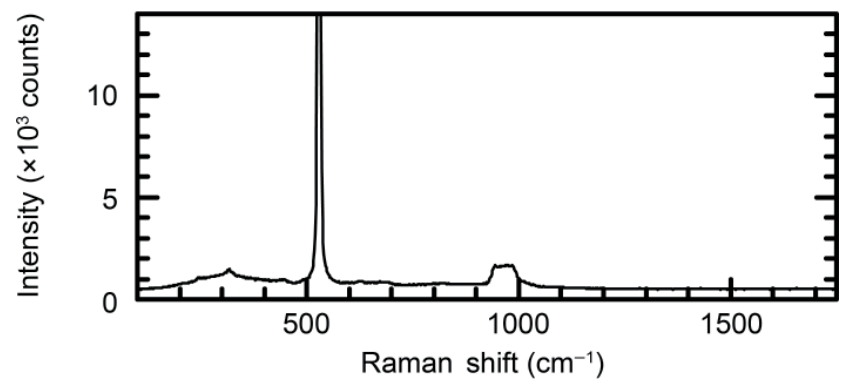

(a)

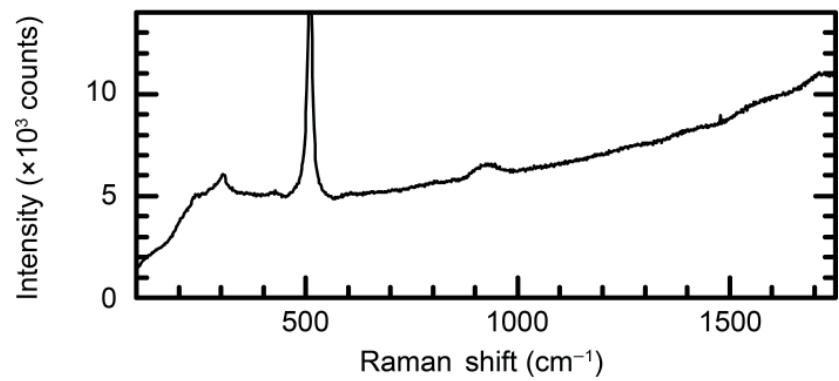

(b)

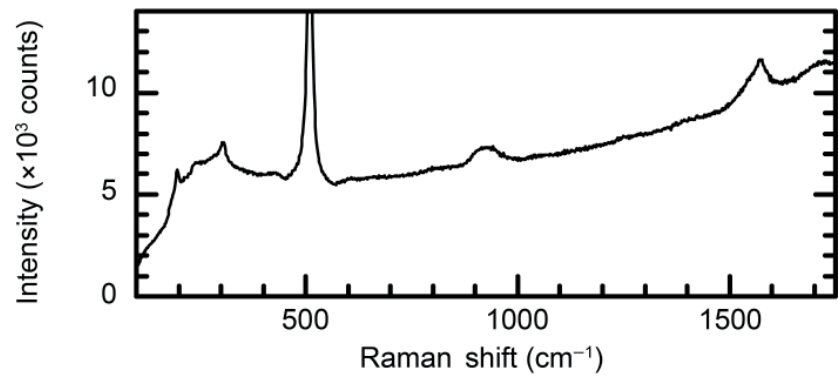

(c)

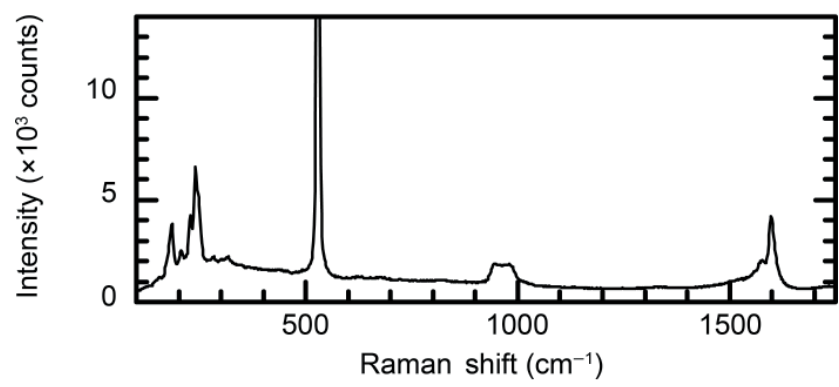

(d)

Figure 3 Raman spectra, pre- and post-growth at $875^{\circ} \mathrm{C}$ : (a) Room temperature, pre-growth; (b) $875{ }^{\circ} \mathrm{C}$, pre-growth; (c) $875{ }^{\circ} \mathrm{C}$, postgrowth; (d) room temperature, post-growth. All spectra are plotted on the same scale 
the blackbody signal is appreciable in the $\mathrm{D}$ and $\mathrm{G}$ band areas as shown in Fig. 3(b) (pregrowth, $875^{\circ} \mathrm{C}$ ). Still, the post-growth Raman signal for the $\mathrm{G}$ band is easily distinguished from the blackbody background. The D-band signal is much weaker for growth at this higher temperature, and it cannot be seennot because it is obscured by blackbody radiation -but mainly because the D-G ratio is much lower for growth at this temperature. In Figs. 3(b) and 3(c) there is one RBM visible. Again, upon cooling to room temperature (Fig. 3(d)), more RBMs are seen, and their intensity is much higher. Here again, a clear G- peak is observed at room temperature which is not distinguishable at high temperature.

Figure 4 shows SEM images from these samples after growth. Figure 4(a) shows the 725 ${ }^{\circ} \mathrm{C}$ grown sample, using $5 \mathrm{kV}$ acceleration voltage and the upper detector of a Hitachi S-4700 SEM. Thick nanotubes are abundant, as well as some narrow SWNT candidates. Nanoparticles with sub $10 \mathrm{~nm}$ widths are also readily seen all across the surface. Figure $4(\mathrm{~b})$ shows the $875{ }^{\circ} \mathrm{C}$ grown sample imaged using $5 \mathrm{kV}$ acceleration voltage. The thick nanotubes are absent and thin nanotubes and bundles of nanotubes are visible at fairly low densities. Nanoparticles of similar scale to the lowertemperature growth are also readily detected. Figure $4(\mathrm{c})$ is a lower $1 \mathrm{kV}$ acceleration voltage image of the high temperature grown sample. White lines can be seen criss-crossing the surface of the substrate. These are almost certainly due to the charging effect of surface nanotubes, which are difficult to detect in geometrical contrast at $5 \mathrm{kV}$ acceleration voltage.

We now turn to the growth dynamics. Timed series of Raman spectra are shown for the lowtemperature growth in Fig. 5 and the hightemperature growth in Fig. 6. Spectra were taken at $5 \mathrm{~s}$ intervals with $5 \mathrm{~s}$ integration times during the CVD process. The series run from bottom to top and are offset by a fixed constant for each successive time step. Only the RBM and D-G regions are shown. For the low-temperature growth, the $\mathrm{G}$ band evolution can be traced most clearly from non-existent, to increasing, to saturated. Although quite a bit lower, both the D band and RBM can be traced and their growth parallels that of the $\mathrm{G}$ band.
The high-temperature growth (Fig. 6) is similar but the evolution from growth to saturation occurs over a longer time interval. On this scale two RBMs can be seen, one of which is relatively clear and dominant, as well as a weaker shoulder. Their growth parallels the $\mathrm{G}$ band very closely. The D band is too weak to track.

Greater insight into the growth process can be obtained by plotting the Raman band intensities as a function of time. To do this meaningfully it
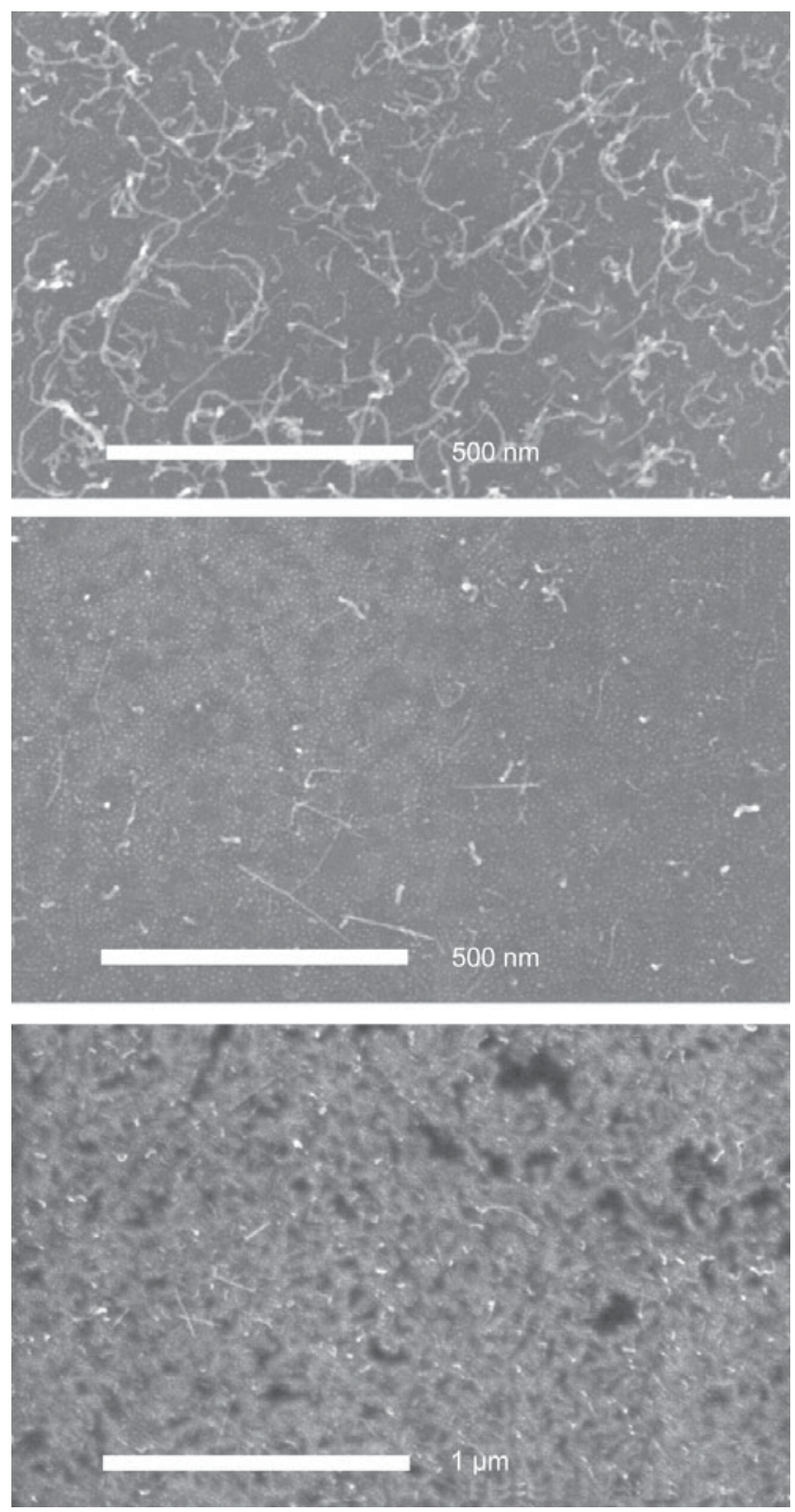

Figure 4 Scanning electron microscope images of sample grown (a) at $725^{\circ} \mathrm{C}$ using $5 \mathrm{kV}$ acceleration voltage, (b) at $875{ }^{\circ} \mathrm{C}$ using 5 $\mathrm{kV}$ acceleration voltage, and (c) at $875^{\circ} \mathrm{C}$ using $1 \mathrm{kV}$ acceleration voltage 


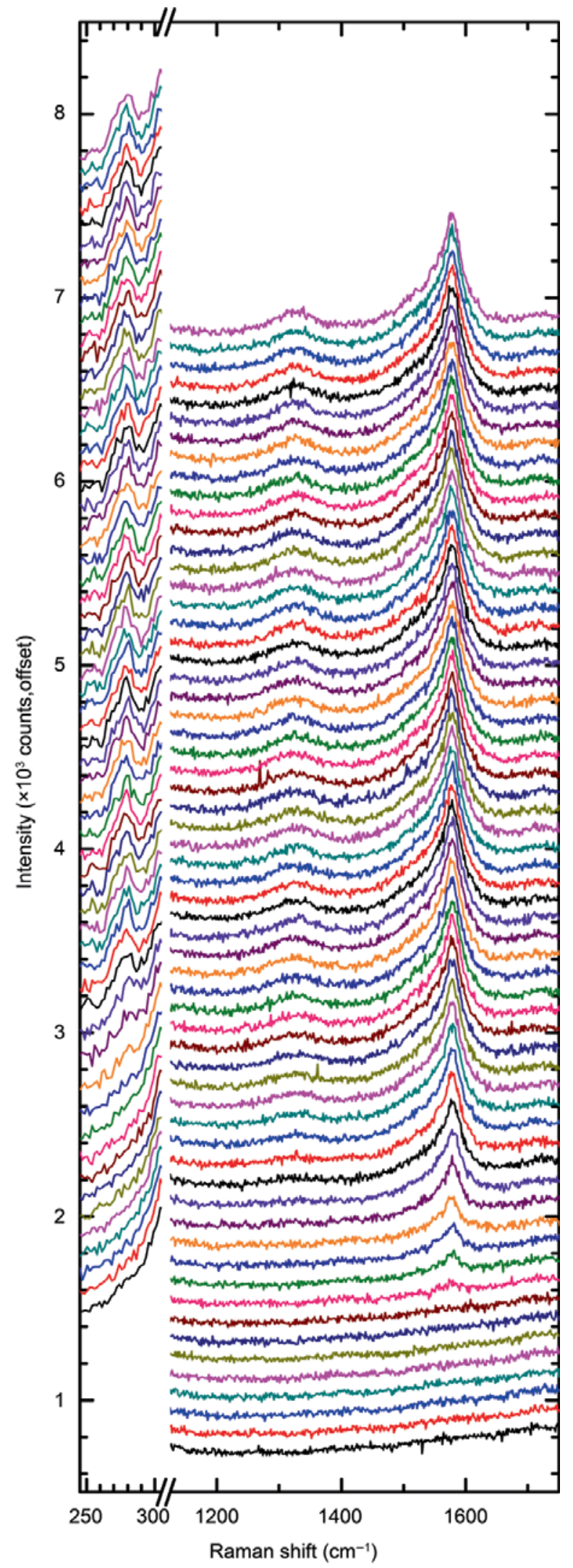

Figure 5 Raman movie for growth at $725^{\circ} \mathrm{C}$. Raman spectra were taken at $5 \mathrm{~s}$ intervals using $5 \mathrm{~s}$ integration times. Successive frames run from bottom to top

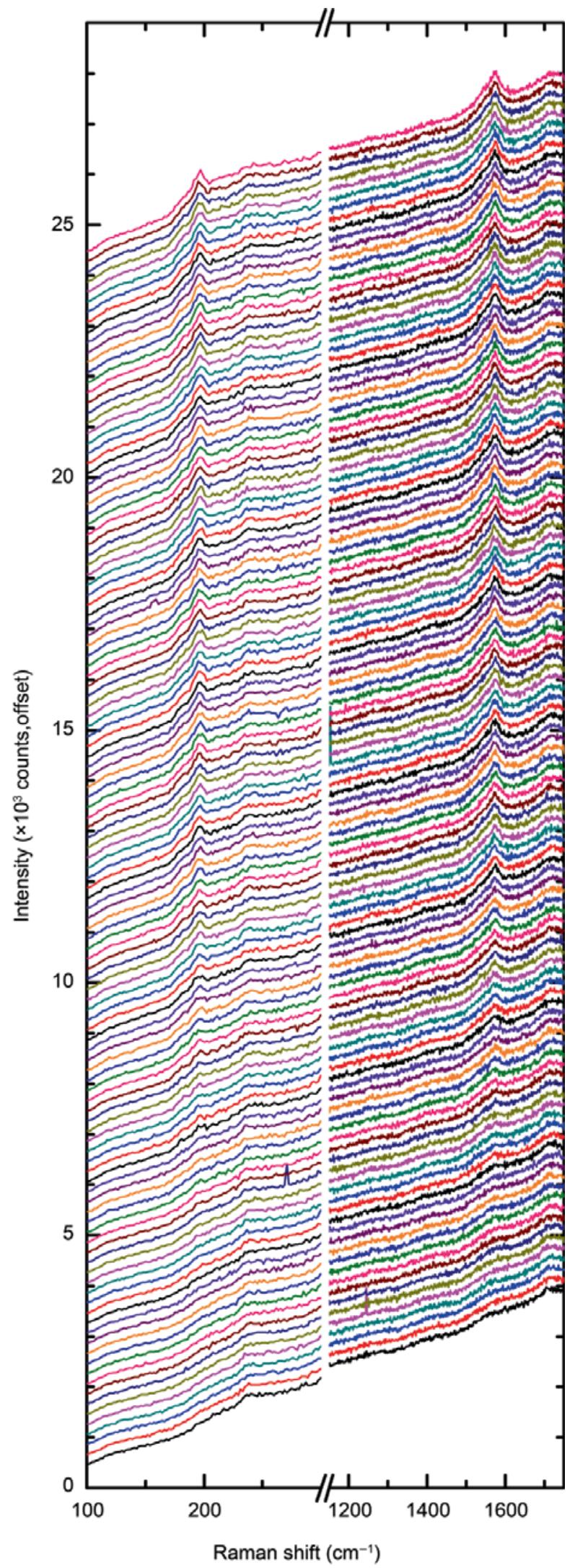

Figure 6 Raman movie for growth at $875^{\circ} \mathrm{C}$. Raman spectra were taken at $5 \mathrm{~s}$ intervals using $5 \mathrm{~s}$ integration times. Successive frames run from bottom to top 
is essential to subtract the background correctly. Figure 7 illustrates our approach to background subtraction. The background was determined by drawing a straight line between two points on either side of the Raman band of interest. These points were determined by averaging the position and intensity of five to seven data points which lay clearly on either side of the Raman band. It is important to realize the blackbody signal can evolve during growth, and other features which overlap with the Raman band of interest may also be changing. If the background is not subtracted properly the evolution one observes is not just that of the particular Raman band of interest, but also any other changes which are superimposed. For example, at high temperature $T$ even a very small change in temperature can cause a large fluctuation in the background, since the integrated blackbody intensity scales as $T^{4}$. Furthermore, as growth proceeds the emissivity of the sample can change, causing the blackbody signal to evolve.

Figure 8 shows the evolution of all the bands for the low-temperature growth. Here the final intensity of each band is scaled to the $G$ band final intensity. The $G$ band shows an exponential onset, a linear growth phase, and an exponentially decaying saturation phase. The D and G bands are weak and so the data are noisy, but the scaled bands overlap very well with the $G$ band, and are basically indistinguishable from the $\mathrm{G}$ band to within experimental uncertainty, though we cannot rule out - given the experimental uncertainty - that the D

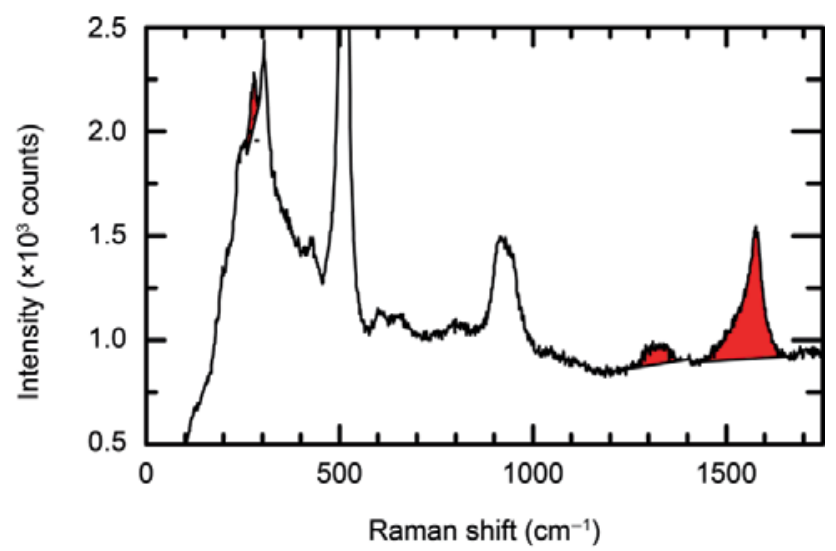

Figure 7 The integrated, background subtracted intensities of RBM, $D$, and $G$ bands (areas in red, from left to right) were extracted for each frame. This example is a frame from the movie taken at $725^{\circ} \mathrm{C}$ band may lag slightly behind the G band.

Figure 9 shows the scaled evolution of the $\mathrm{G}$ and RBM bands for the high-temperature growth. The overlap is remarkable. The D band is too weak to obtain any meaningful data. For the $G$ band and RBM bands there is an initial exponential increase, a linear growth phase and a saturation phase and they

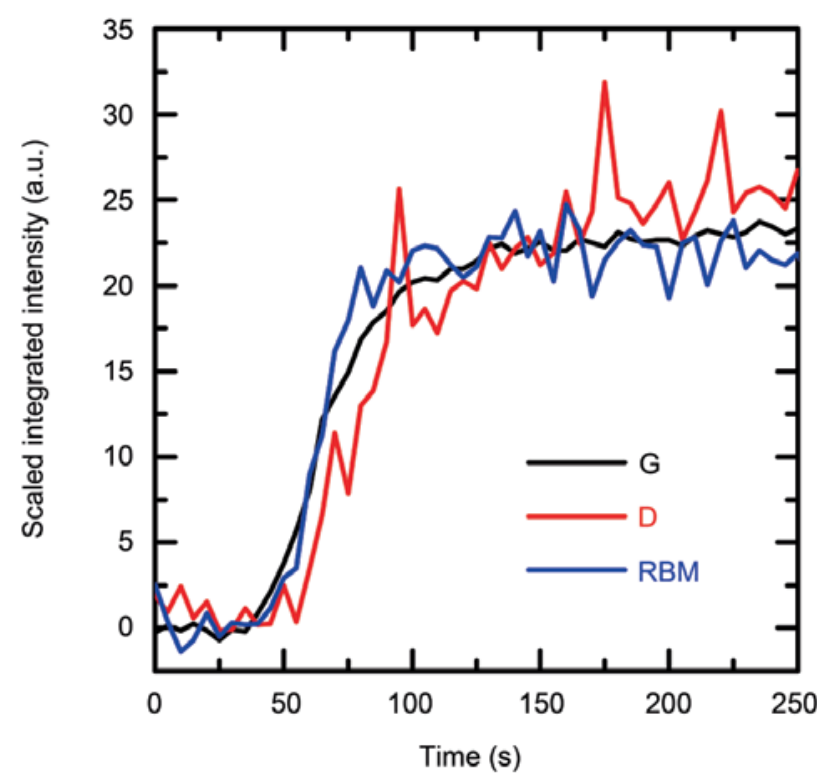

Figure 8 Evolution of the integrated intensities of G, D, and RBM during growth at $725^{\circ} \mathrm{C}$. Bands are scaled to the $\mathrm{G}$ band final intensity

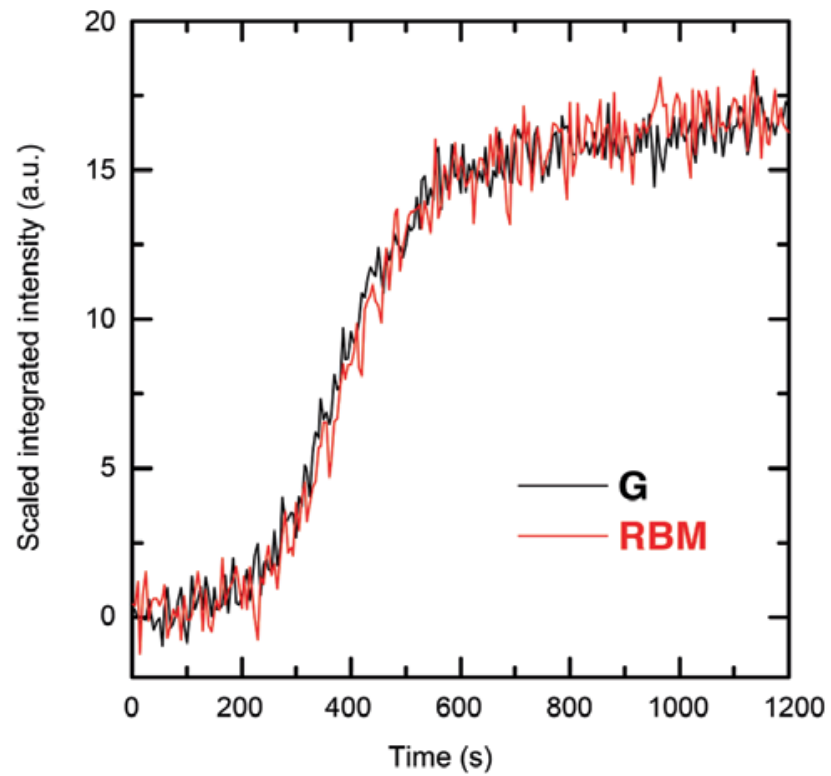

Figure 9 Evolution of the integrated intensities for $G$ and RBM bands for growth at $875^{\circ} \mathrm{C}$. The RBM final intensity is scaled to the $\mathrm{G}$ band. The $\mathrm{D}$ band is too low to track 
track each other remarkably well.

Given that this involves sampling over a vast number of nanotubes this may have important implications. The RBM evolution, which represents only one specific diameter SWNT here, exactly tracks the $G$ band evolution, which represents a large number of diameters, and would even include non-SWNT $\mathrm{sp}^{2}$ carbon were it present in sufficient abundance to produce an appreciable signal. If one particular species of nanotube tracks the $G$ band, it is probable that all nanotubes follow the $G$ band. Thus, the data are evidence - albeit indirect - that, at least for this particular type of CVD, there is no net evolution of the SWNT distribution (i.e., diameter or chirality) during growth. Essentially, the population that you start with is the population you end up with.

The onset of the Raman signal shows an accelerating growth phase which is apparently associated with the nucleation and initial lengthening of SWNTs. This is followed by a linear phase likely due to steady lengthening, and then a saturation phase which represents the termination process (Fig. 10). Interestingly, here the acceleration and termination timescales are both the same order $(\sim 10 \mathrm{~s}$ at $725^{\circ} \mathrm{C}, \sim 100 \mathrm{~s}$ at $875^{\circ} \mathrm{C}$ ) which suggests a possible common origin for the two processes. Exponential increases in Raman signal during nucleation might come from the number of catalyst nanoparticles

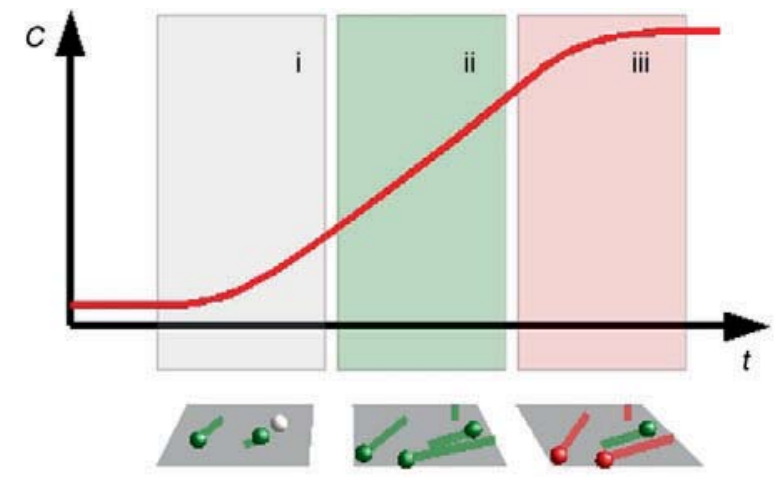

Figure 10 Schematic of growth evolution. The mass of carbon (C) increases exponentially with time $(t)$ in an initial "acceleration" phase associated with nucleation and lengthening (i). Next is a period of relatively steady growth (ii). Finally, there is an exponential deactivation process (iii). Nanoparticles that are bare are white, those extruding nanotubes are green, and those that have terminated are red. Growing nanotubes are green and completed nanotubes are red becoming active exponentially, or it might come from nucleated nanotubes growing initially at an exponential rate.

We next compare these data from other data obtained from in situ experiments. Though we intentionally chose a catalyst and conditions to obtain a low yield of surface SWNTs, it is instructive to compare with the growing number of in situ studies of vertically aligned forest growth. In these studies, average nanotube growth rates can be inferred from forest heights as a function of time.

By monitoring the thickness of nanotube forest films made by acetylene CVD by Fabry-Perot reflectivity [6,7], Puretzky et al. found an initially accelerating growth rate and pointed out that it takes longer time to reach the maximum growth rate at higher temperature [7]. This is similar to what is observed here. In earlier plasma CVD experiments by Kim et al. using similar methods, an initial increase in growth rate with time was seen, and this was attributed to a surface diffusion process [5]. By measuring optical absorbance of forest films made using ethanol CVD, Einarsson et al. found a simple exponential decay of the growth rate, although no non-linear onset was seen in that case [8].

By measuring the displacement of a reflected laser beam, Mershot et al. found an initial growth transient, followed by relatively steady growth and a sudden termination. The initial transient was attributed to gas mixing in that case [9]. This raises a point worth emphasizing, namely, that the initial nucleation phase of CVD growth in particular will be affected by the specifics of the transition from purge gas to carbon precursor. This is a necessary step in typical nanotube CVD processes. In our case any gas concentration transient is not well characterized; however, we believe that it is likely to be short. Given that the reactor is only $7 \mathrm{~mm}$ in diameter, the gas in the hot zone should cycle completely within about $30 \mathrm{~s}$. In video studies of forest height at low presssures, Puretzky et al. observed an initial slow growth attributed either to the travel time of source gases in the reactor, or to initially slow nucleation and alignment [10]. They also showed steady growth and sudden termination phases. In video movies of acetylene forest growth Mattevi et al. saw both 
steady growth with sudden termination, as well as initial acceleration and subsequent deceleration [11]. In similar ethanol CVD video studies we only saw exponential growth rate decay, though the height resolution may simply not have been sufficient to observe any initial exponential onset [12].

Other classes of experiments have also revealed similar evolution curves. Single SWNT growth rates have also been measured by TEM, and similar initial acceleration, steady growth, and termination phases have all been reported [13]. An initial acceleration phase has also been reported for nanotubes in microbalance experiments which measure the mass of grown material as a function of growth time [14].

To compare more directly with other in situ Raman studies, a roughly linearly increasing $G$ band with time was observed by Chiashi et al. [1]. However, the time resolution was lower, growth was halted before saturation was observed, and other bands were not visible in situ. Here we do see the G band increase, but have a more complicated growth curve with distinct acceleration, steady growth, and saturation phases. We obtained qualitatively similar G and D band evolution curves for low-temperature MWNT growth in a previous work [2]. This was despite over $100 \times$ higher flow rates in that case, and an entirely different reactor design.

Dittmer et al. used ethylene as a precursor and took D, G and RBM snapshots in situ, although this was after cooling to room temperature for various growth durations [3]. The RBM density was found to increase, and a subtle shift of RBM to large diameter nanotubes was suggested. We did not see evidence for such a shift. That difference might possibly be attributable to their use of a focused spot size compared to our larger sampling area. At the same time, because RBMs are more difficult to detect at high-temperatures, we were not able to see the many RBMs that are readily detected at room temperature so the comparison is difficult. Dittmer et al. also saw a very large evolution in D/G ratio, with it first decreasing and then increasing by an order of magnitude. In our case no such change was detected with $G, D$, and even RBM bands tracking each other, at least to a fairly good approximation.

Picher et al. tracked the $G$ band as a function of time, temperature, and partial pressure. They fitted the growth rate to the exponentially decaying growth model, which is qualitatively similar to the decaying growth rate we see here over longer times, although in their case no initial acceleration phase was reported. The temperature scaling of the initial growth rate and $\mathrm{D} / \mathrm{G}$ ratio in that work are generally consistent with our results from video movies of forest growth [12]. Although not discussed in Ref. [4], the somewhat surprising result that the high-temperature run shows a slower evolution than the low-temperature run in our experiments (e.g., compare time scales for Fig. 9 vs Fig. 8) is consistent with initial growth rates reported in their supplementary data. We speculate that this slow evolution at high temperature might be the result of the desorption of carbon, which would decrease the initial growth rate at higher temperatures. The question of how the various bands track each other was not specifically investigated by Picher et al., although in that work the RBM and D band can be seen to increase along with the G band, which is at least consistent with the scaling we observe.

\section{Conclusions}

In situ Raman spectroscopy is maturing from being an experimental technique to becoming an established technique for monitoring synthesis in real time, as it happens, and allowing us to probe the CVD growth process in detail. Further insight into nucleation, steady growth and termination phases can be expected from in situ Raman studies in the near future. These and other in situ methods will clarify our understanding of nanotube synthesis, allow for the rigorous testing of theoretical models of growth, and ultimately contribute to our effort to achieve precision control over the end product of the nanotube CVD synthesis process.

\section{Acknowledgements}

A discovery grant (PF, AL) from Natural Sciences and Engineering Research Council of Canada (NSERC) and a grant (AL) from Le Fonds québécois de la recherche sur la nature et les technologies 
(FQRNT) are gratefully acknowledged. We are grateful for the ongoing assistance of P. Marshall, Hue Tran, Jeff Fraser and other IMS staff and for preliminary research in this area by Jeffery Bond and Kate Kaminska. We are grateful for the funding of in situ Raman studies via the former JST-CREST "Nanofactory" project led by Yoshikazu Homma.

\section{References}

[1] Chiashi, S.; Murakami, Y.; Miyauchi, Y.; Maruyama S. Cold wall CVD generation of single-walled carbon nanotubes and in situ Raman scattering measurements of the growth stage. Chem. Phys. Lett. 2004, 386, 8991.

[2] Kaminska, K.; Lefebvre, J.; Austing, D. G.; Finnie, P. Realtime in situ Raman imaging of carbon nanotube growth. Nanotechnology 2007, 18, 165707.

[3] Dittmer, S.; Olofsson, N.; Ek Weis, J.; Nerushev, O. A.; Gromov, A. V.; Campbell, E. E. B. In situ Raman studies of single-walled carbon nanotubes grown by local catalyst heating. Chem. Phys. Lett. 2008, 457, 206-210.

[4] Picher, M.; Anglaret, E.; Arenal, R.; Jourdain, V. Selfdeactivation of single-walled carbon nanotube growth studied by in situ Raman measurements. Nano Lett. 2009, 9, 542-547.

[5] Kim, D.; Jang, H. -S.; Kim, C. -D.; Cho, D. -S.; Yang, H. -S.; Kang, H. -D.; Min, B. -K.; Lee, H. -R. Dynamic growth rate behavior of a carbon nanotube forest characterized by in situ optical growth monitoring. Nano Lett. 2003, 3, 863-865.

[6] Geohegan, D. B.; Puretzky, A. A.; Ivanov, I. N.; Jesse, S.; Eres, G. In situ growth rate measurements and length control during chemical vapor deposition of vertically aligned multiwall carbon nanotubes. Appl. Phys. Lett. 2003, 83, 1851-1853.

[7] Puretzky, A. A.; Geohegan, D. B.; Jesse, S.; Ivanov, I. N.;
Eres, G. In situ measurements and modeling of carbon nanotube array growth kinetics during chemical vapor deposition. Appl. Phys. A 2005, 81, 223-240.

[8] Einarsson, E.; Murakami, Y.; Kadowaki, M.; Maruyama, S. Growth dynamics of vertically aligned single-walled carbon nanotubes from in situ measurements. Carbon 2008, 46, 923-930.

[9] Meshot, E. R.; Hart, A. J. Abrupt self-termination of vertically aligned carbon nanotube growth. Appl. Phys. Lett. 2008, 92, 113107.

[10] Puretzky, A. A.; Eres, G.; Rouleau, C. M.; Ivanov, I. N.; Geohegan, D. B. Real-time imaging of vertically aligned carbon nanotube array growth kinetics. Nanotechnology 2008, 19, 055605.

[11] Mattevi, C.; Wirth, C.; Hofmann, S.; Blume, R.; Cantoro, M.; Ducati, C.; Cepek, C.; Knop-Gericke, A.; Milne, S.; Castellarin-Cudia, C.; Dolafi, S.; Goldoni, A.; Schloegl, R.; Robertson, J. In-situ photoelectron spectroscopy study of catalyst-support interactions and growth of carbon nanotube forests. J. Phys. Chem. C 2008, 112, $12207-$ 12213.

[12]Vinten, P.; Lefebvre, J.; Finnie, P. Kinetic critical temperature and optimized chemical vapor deposition growth of carbon nanotubes. Chem. Phys. Lett., 2009, 469, 293-297

[13] Lin, M.; Tan, J. P. Y.; Boothroyd, C.; Loh, K.; Tok, E. S.; Foo, Y. -L. Direct observation of single-walled carbon nanotube growth at the atomistic scale. Nano Lett. 2006, 6, 449-452

[14] Valiente, A.; Lopez, P.; Ramos, R.; Ruiz, A.; Li, C.; Xin, Q. In situ study of carbon nanotube formation by $\mathrm{C}_{2} \mathrm{H}_{2}$ decomposition on an iron-based catalyst. Carbon 2000, 38, 2003-2006

[15] Kaminska, K.; Lefebvre, J.; Austing, D. G.; Finnie, P. Realtime global Raman imaging and optical manipulation of suspended carbon nanotubes. Phys. Rev. B 2006, 73, 235410 